Volume 131, Number 2, Pages 351-357

S 0002-9939(02)06558-9

Article electronically published on June 5, 2002

\title{
CORRECTION TO THE PAPER "DUALITY AND FLAT BASE CHANGE ON FORMAL SCHEMES"
}

\author{
LEOVIGILDO ALONSO TARRÍO, ANA JEREMÍAS LÓPEZ, AND JOSEPH LIPMAN
}

(Communicated by Wolmer V. Vasconcelos)

\begin{abstract}
In $\S 8.3$ of our paper "Duality and Flat Base Change on Formal Schemes" some important results concerning localization and preservation of coherence by basic duality functors were based on the false statement that any closed formal subscheme of an open subscheme of the completion $\mathcal{P}$ of a relative projective space is an open subscheme of a closed formal subscheme of $\mathcal{P}$. In this note, the said results are provided with solid foundations.
\end{abstract}

In Proposition 8.3.1 of our paper [DFS], the duality functors $f^{!}$and $f^{\#}$ associated to a pseudo-proper map $f: X \rightarrow Y$ of noetherian formal schemes (i.e., right adjoints of suitable restrictions of the derived direct-image functor $\mathbf{R} f_{*}$ ) are asserted to be local on $\mathcal{X}$, as a consequence of flat base change. Moreover, in Proposition 8.3.2 it is asserted that (roughly speaking) $f^{\#}$ preserves coherence. Brian Conrad pointed out that our justifications are deficient because they use the claim 8.3.1(c) that a map between noetherian formal schemes that can be factored as a closed immersion followed by an open one can also be factored as an open immersion followed by a closed one, which is not true in general 1 Indeed, Conrad observed that for any $(A, x, p)$ with $A$ an adic domain, $x \in A$ such that $B:=A_{\{x\}}$ is a domain, and $p$ a nonzero $B$-ideal contracting to $(0)$ in $A$, the natural map $\operatorname{Spf}(B / p) \rightarrow \operatorname{Spf}(A)$ is a counterexample. Such a triple was provided to us by Bill Heinzer:

With $w, x, y, z$ indeterminates over a field $k$, set

$$
A:=k[w, x, z][[y]] \text { and } B:=A_{\{x\}}=k[w, x, 1 / x, z][[y]] .
$$

Let $P$ be the prime ideal $(w, z) A$ and $R:=A_{P} \subset B_{P B}=: S$, so that $R \subset S$ are twodimensional regular local domains such that the residue field of $S$ (i.e., the fraction field of $k[x, 1 / x][[y]]$ ) is transcendental over that of $R$ (i.e., the fraction field of $k[x][[y]])$. Then $[\mathrm{HR}$. p. 364, Theorem 1.12] says that there exist infinitely many height-one prime $S$-ideals in the generic fiber over $R$. Any of these contracts in $B$ to a (prime) $p$ as above.

Received by the editors July 2, 2001 and, in revised form, September 7, 2001.

2000 Mathematics Subject Classification. Primary 14F99; Secondary 13D99, 14B15, 32C37.

Key words and phrases. Grothendieck duality, formal scheme.

The first two authors were partially supported by Spain's DGESIC PB97-0530 research project. They thank the Mathematics Department of Purdue University for its hospitality and support.

The third author was partially supported by the National Security Agency.

${ }^{1}$ The "proof" breaks down in the second-last line of [DFS] p. 88], where it is erroneously stated that localization followed by completion commutes with forming kernels of homomorphisms of adic rings. 
Our purpose here is to validate the aforementioned Propositions by means of a "localization" Lemma (1) below). Thus all other results in DFS depending on these Propositions remain as they are. (No other results depend on the faulty 8.3.1(c).)

However, Proposition 8.3.1 is weakened in that we get isomorphisms which are not a priori functorial or canonical at the level of derived categories but only at the level of homology sheaves. This drawback does not affect the applications.

(Derived functoriality and canonicity might well be attainable, for example through a suitable variant - if such exists - of compactification of separated pseudofinite type maps of noetherian formal schemes.)

Notation and terminology are as in [DFS] (which has an index starting on p. 125). For example, with $\mathcal{I}_{y}$ an ideal of definition of the noetherian formal scheme $y$ and $\Gamma_{y}^{\prime}(-)$ the torsion functor $\varliminf_{n} \mathcal{H o m}\left(\mathcal{O}_{y} / \mathcal{I}_{y}^{n},-\right)$, an $\mathcal{O}_{y}$ - complex $\mathcal{F}$ lies in $\widetilde{\mathbf{D}}_{\mathrm{qc}}^{+}(\mathrm{y})$ if the homology $H^{i}(\mathcal{F})$ vanishes for $i \ll 0$ and the derived-torsion complex $\mathbf{R} \Gamma_{y}^{\prime} \mathcal{F}$ has quasi-coherent homology. In particular, $\mathbf{D}_{\mathrm{qc}}^{+}(y) \subset \widetilde{\mathbf{D}}_{\mathrm{qc}}^{+}(y)$ DFS, p. 54, 5.2.10].

Lemma 1. Let

$$
\mathcal{U} \stackrel{q_{k}}{\longrightarrow} \mathcal{V}_{k} \stackrel{h_{k}}{\longrightarrow} x_{k} \stackrel{f_{k}}{\longrightarrow} y \quad(k=1,2)
$$

be maps of noetherian formal schemes with $q_{k}$ a closed immersion, $h_{k}$ an open immersion, $f_{k}$ pseudo-proper, and $f_{1} h_{1} q_{1}=f_{2} h_{2} q_{2}$. Then one can define isomorphisms

$$
q_{1}^{!} h_{1}^{*} f_{1}^{!} \mathcal{F} \stackrel{\sim}{\longrightarrow} q_{2}^{!} h_{2}^{*} f_{2}^{!} \mathcal{F}, \quad q_{1}^{\#} h_{1}^{*} f_{1}^{\#} \mathcal{F} \stackrel{\sim}{\longrightarrow} q_{2}^{\#} h_{2}^{*} f_{2}^{\#} \mathcal{F} \quad\left(\mathcal{F} \in \widetilde{\mathbf{D}}_{\mathrm{qc}}^{+}(\mathrm{y})\right)
$$

such that the induced homology isomorphisms are canonical and functorial.

Proof. Recall that $f^{!}$is the notation used for $f_{\mathrm{t}}^{\times}$when $f$ is pseudo-proper, and that $\boldsymbol{\Lambda}_{X}(-):=\mathbf{R} \mathcal{H o m}^{\bullet}\left(\mathbf{R} \Gamma_{X}^{\prime} \mathcal{O}_{X},-\right)$. The isomorphisms of functors from $\mathbf{D}(y)$ to $\mathbf{D}(\mathcal{U})$,

$$
\boldsymbol{\Lambda}_{\mathcal{U}} q_{k}^{!} h_{k}^{*} f_{k}^{!} \stackrel{\sim}{\longrightarrow} \boldsymbol{\Lambda}_{\mathcal{U}} q_{k}^{!} \boldsymbol{\Lambda}_{\mathcal{V}_{k}} h_{k}^{*} f_{k}^{!} \stackrel{\sim}{\longrightarrow} \boldsymbol{\Lambda}_{\mathcal{U}} q_{k}^{!} h_{k}^{*} \boldsymbol{\Lambda}_{X_{k}} f_{k}^{!} \stackrel{\sim}{\longrightarrow} q_{k}^{\#} h_{k}^{*} f_{k}^{\#} \quad(k \in\{1,2\})
$$

where the second is obvious and the other two are given by [DFS, Corollary 6.1.5], show that it suffices to establish the first isomorphism in Lemma 1.

Let $\mathcal{I}_{y} \subset \mathcal{O}_{y}, \mathcal{I}_{x_{1}} \subset \mathcal{O}_{x_{1}}$ and $\mathcal{I}_{x_{2}} \subset \mathcal{O}_{x_{2}}$ be ideals of definition such that $\mathcal{I}_{y} \mathcal{O}_{X_{1}} \subset \mathcal{I}_{X_{1}}$ and $\mathcal{I}_{y} \mathcal{O}_{X_{2}} \subset \mathcal{I}_{X_{2}}$. The ideals $\mathcal{I}_{1}:=\mathcal{I}_{X_{1}} \mathcal{O}_{u}, \mathcal{I}_{2}:=\mathcal{I}_{X_{2}} \mathcal{O}_{u}$ and $\mathcal{I}:=\mathcal{I}_{1}+\mathcal{I}_{2}$ are ideals of definition of the formal scheme $\mathcal{U}$, possibly different. For each $n>0$ let $u_{n}: U_{n} \rightarrow \mathcal{U}$ be the closed immersion determined by $\mathcal{I}^{n}$ (so that $U_{n}$ is an ordinary noetherian scheme with the same underlying topological space as $\mathcal{U}$, but with structure sheaf $\left.\mathcal{O}_{\mathcal{U}} / \mathcal{I}^{n}\right)$. The desired isomorphism results from the existence - to be shown - of a family of isomorphisms

$$
u_{n *} u_{n}^{!} q_{1}^{!} h_{1}^{*} f_{1}^{!} \mathcal{F} \stackrel{\sim}{\longrightarrow} u_{n *} u_{n}^{!} q_{2}^{!} h_{2}^{*} f_{2}^{!} \mathcal{F} \quad\left(n>0, \mathcal{F} \in \widetilde{\mathbf{D}}_{\mathrm{qc}}^{+}(\mathrm{y})\right),
$$

compatible with the homotopy colimit triangles given by DFS, Lemma 5.4.1, Proposition 5.2.1(a), and Example 6.1.3(4)], for $k \in\{1,2\}$ :

$$
\bigoplus_{n>0} u_{n *} u_{n}^{!} q_{k}^{!} h_{k}^{*} f_{k}^{!} \mathcal{F} \longrightarrow \bigoplus_{n>0} u_{n *} u_{n}^{!} q_{k}^{!} h_{k}^{*} f_{k}^{!} \mathcal{F} \longrightarrow q_{k}^{!} h_{k}^{*} f_{k}^{!} \mathcal{F} \stackrel{+}{\longrightarrow} .
$$

For, a basic property of triangles is that such a family of isomorphisms extends (not necessarily uniquely!) to an isomorphism between the "summits" $q_{k}^{!} h_{k}^{*} f_{k}^{!} \mathcal{F}$. 
Although the isomorphisms (11) will be canonical, it does not follow that their extension to the summits is. However the $i$-th homology $H^{i}\left(q_{k}^{!} h_{k}^{*} f_{k}^{!} \mathcal{F}\right)$ is canonically isomorphic to the direct limit of $H^{i}\left(u_{n *} u_{n}^{!} q_{k}^{!} h_{k}^{*} f_{k}^{!} \mathcal{F}\right)$, and so we will have produced canonical functorial homology isomorphisms

$$
H^{i}\left(q_{1}^{!} h_{1}^{*} f_{1}^{!} \mathcal{F}\right) \stackrel{\sim}{\longrightarrow} H^{i}\left(q_{2}^{!} h_{2}^{*} f_{2}^{!} \mathcal{F}\right) \quad(i \in \mathbb{Z}) .
$$

Digression. The definition of (1) is based on the fact - well-known, though not yet conveniently packaged in full generality and detail - that on the category of separated finite-type maps of arbitrary noetherian schemes, there is an essentially unique pseudofunctor! taking values in $\mathbf{D}_{\mathrm{qc}}^{+}$, restricting to the duality pseudofunctor $f^{!}$on the subcategory of proper maps $f$ and to $f^{*}$ on the subcategory of open immersions, 2 and compatible with open base change in the sense that for any noetherian-scheme map

$$
g=f h^{\prime}=h f^{\prime}: X \rightarrow T
$$

with $h, h^{\prime}$ open immersions and $f, f^{\prime}$ proper - so that in the associated noetherianscheme diagram

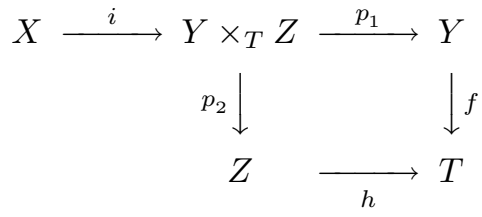

(with $p_{1}, p_{2}$ the canonical projections, $p_{1} i=h^{\prime}, p_{2} i=f^{\prime}$ ) $i$ is an open and closed immersion - the following natural diagram of isomorphisms commutes:

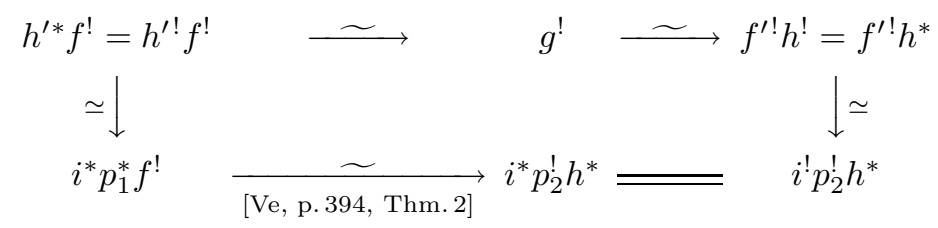

For the construction of ! see [De p. 318, Prop. 3.3.4]. To verify the hypotheses there, one needs Nagata's theorem that any finite-type separable map of noetherian schemes admits a factorization of the form $f h^{\prime}$ as above; and one needs to prove the cited Theorem 2 in [Ve] without assuming finite dimensionality of the schemes involved. For the former, see [Lü] and [Co. For the latter, see $[\mathrm{Ne}, \S 6]$ (keeping in mind the equivalence of categories given by $\mathrm{Ha}$ p. 47, Prop.4.8]), which treats the case - sufficient for present purposes - of open base change, or the outline for proving the general case [Li, p. 120, Cor. (4.3)].

The above-mentioned lack of canonicity obstructs immediate extension of the pseudofunctor! from ordinary to formal schemes, an extension whose existence would give a stronger canonical version of Lemma 1-as! already does for ordinary noetherian schemes. (Nevertheless such an extension might always exist for reasons as yet unknown to us.)

\footnotetext{
${ }^{2}$ There is no conflict when $f$ is both proper and an open immersion (i.e., $f$ is an open and closed immersion) because then $f^{*}$ is right-adjoint to $f_{*}$, so one can identify $f^{*}$ and the dualizing $f^{!}$.
} 
Let us return to the proof. The isomorphisms (1) arise from applying $u_{n *}$ to the below isomorphisms (4), that we describe next. Consider the diagram

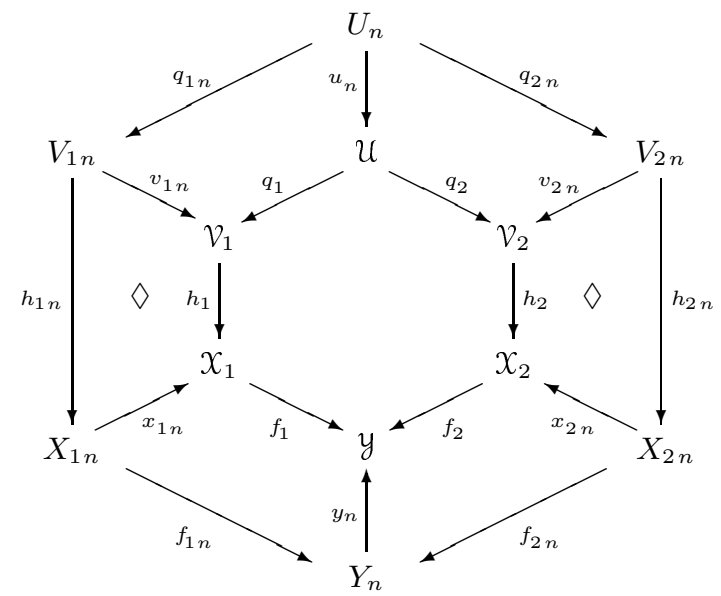

where $x_{1 n}, x_{2 n}, y_{n}$ are the closed immersions given by the ideals $\mathcal{I}_{X_{1}}^{n}, \mathcal{I}_{X_{2}}^{n}$ and $\mathcal{I}_{y}^{n}$ respectively, the maps $f_{1 n}$ and $f_{2 n}$ are induced by $f_{1}$ and $f_{2}$ respectively, the subdiagrams marked by $\diamond$ are fiber squares, and $q_{1 n}$ and $q_{2 n}$ are the closed immersions induced by $q_{1}$ and $q_{2}$, respectively. The outer hexagon is then a diagram of ordinary noetherian schemes with $f_{1 n}$ and $f_{2 n}$ proper maps, $h_{1 n}$ and $h_{2 n}$ open immersions and $q_{1 n}$ and $q_{2 n}$ closed immersions.

Use adic flat base change [DFS, Theorem 7.4] and pseudofunctoriality DFS, Theorem 6.1(b)] to obtain the natural composite isomorphism

$$
s_{1 n}(\mathcal{F}): u_{n}^{!} q_{1}^{!} h_{1}^{*} f_{1}^{!} \mathcal{F} \stackrel{\sim}{\longrightarrow} q_{1 n}^{!} v_{1 n}^{!} h_{1}^{*} f_{1}^{!} \mathcal{F} \stackrel{\sim}{\longrightarrow} q_{1 n}^{!} h_{1 n}^{*} x_{1 n}^{!} f_{1}^{!} \mathcal{F} \stackrel{\sim}{\longrightarrow} q_{1 n}^{!} h_{1 n}^{*} f_{1 n}^{!} y_{n}^{!} \mathcal{F}
$$

and analogously,

$$
s_{2 n}(\mathcal{F}): u_{n}^{!} q_{2}^{!} h_{2}^{*} f_{2}^{!} \mathcal{F} \stackrel{\sim}{\longrightarrow} q_{2 n}^{!} h_{2 n}^{*} f_{2 n}^{!} y_{n}^{!} \mathcal{F}
$$

Using the above-described pseudofunctor on ordinary schemes we write $h_{k n}^{*}=h_{k n}^{!}$. Since $y_{n}^{!} \mathcal{F} \in \mathbf{D}_{\mathrm{qc}}^{+}\left(Y_{n}\right)$ [DFS, p. 59, Theorem 6.1] there results a natural isomorphism

$$
r_{n}(\mathcal{F}): q_{1 n}^{!} h_{1 n}^{*} f_{1 n}^{!} y_{n}^{!} \mathcal{F} \stackrel{\sim}{\longrightarrow} q_{2 n}^{!} h_{2 n}^{*} f_{2 n}^{!} y_{n}^{!} \mathcal{F} .
$$

We have then the natural functorial isomorphisms

$$
s_{2 n}(\mathcal{F})^{-1} r_{n}(\mathcal{F}) s_{1 n}(\mathcal{F}): u_{n}^{!} q_{1}^{!} h_{1}^{*} f_{1}^{!} \mathcal{F} \stackrel{\sim}{\sim} u_{n}^{!} q_{2}^{!} h_{2}^{*} f_{2}^{!} \mathcal{F} \quad(n>0)
$$

Still to be shown is that the isomorphisms (11) are compatible with the triangles (2). Let $u_{n+1}^{n}: U_{n} \rightarrow U_{n+1}$ be the natural closed immersion, and let

$$
t_{n}: u_{n *} u_{n}^{!} \cong u_{n+1 *} u_{n+1 *}^{n}\left(u_{n+1}^{n}\right)^{!} u_{n+1}^{!} \rightarrow u_{n+1 *} u_{n+1}^{!}
$$

be the natural map. By the definitions involved, the compatibility in question amounts to commutativity of the following diagram in the category of functors from $\widetilde{\mathbf{D}}_{\mathrm{qc}}^{+}(\mathrm{y})$ to $\mathbf{D}(\mathcal{U})$, where $c_{k n}(k \in\{1,2\})$ is induced by $t_{n}$, and $b_{k n}$ will be 
defined later:

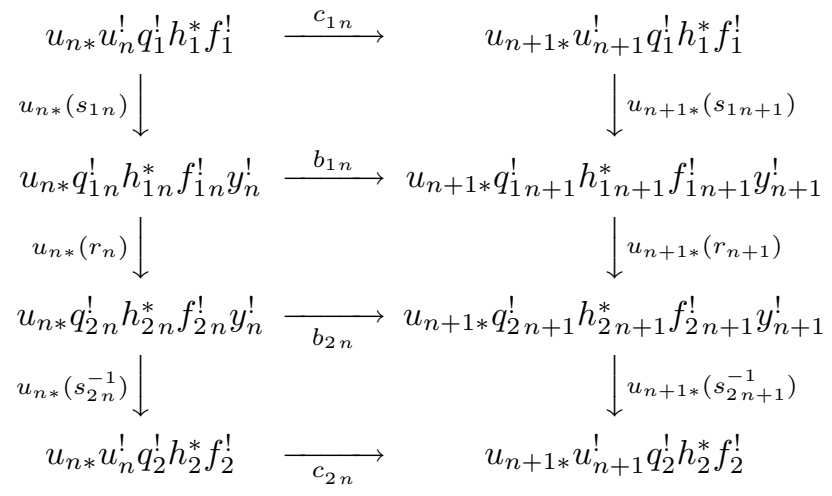

Let us deal first with the top subrectangle of (5). (The bottom one is essentially the same.) To lighten notation, we set $m:=n+1$.

Consider the following expansion of the left side of diagram (3), where all occurrences of "1" in a subscript have been hidden, and where all the vertical arrows represent natural closed immersions, so that for each $\xi \in\{u, v, x, y\}, \xi_{n}=\xi_{m} \circ \xi_{m}^{n}$ :

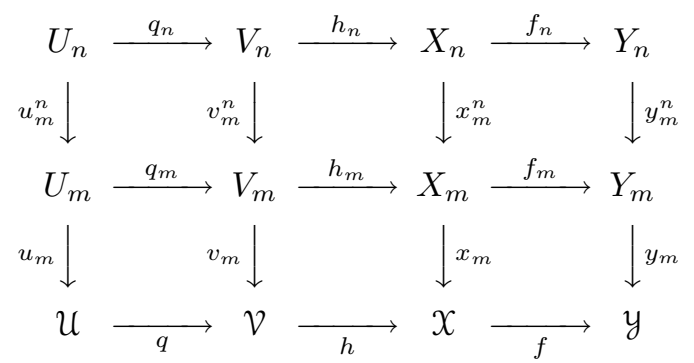

The squares in the middle are fiber squares, to which are associated base-change isomorphisms of the form $h^{*} x^{!} \stackrel{\sim}{\longrightarrow} v^{!} h^{*}$ (with appropriate subscripts attached). With each $\beta$ indicating the use of such a base-change isomorphism, and $a_{n}$ the natural composition

$$
u_{n *} q_{n}^{!} v_{m}^{n !} \stackrel{\sim}{\longrightarrow} u_{m *} u_{m *}^{n} u_{m}^{n !} q_{m}^{!} \rightarrow u_{m *} q_{m}^{!},
$$

one sees then that the top rectangle in (5) expands naturally as



with $b_{1 n}$ in (5) defined to be the composition of the maps in the bottom row. 
It remains only to check commutativity of each of the subrectangles, which is a straightforward exercise requiring only the simplest formal properties of functoriality and pseudofunctoriality 3 except for the subrectangle marked $(*)$, where one uses the transitivity of flat base change [DFS, Lemma 7.5.2(b)].

As for the middle subrectangle in (5), after noting that $u_{n *}=u_{m *} u_{m *}^{n}$ and $y_{n}^{!} \cong y_{m}^{n !} y_{m}^{!}$one can "factor out" $u_{m *}$ and $y_{m}^{!}$, and then use the rather simple duality isomorphism for closed immersions

$$
\operatorname{Hom}_{\mathbf{D}\left(U_{m}\right)}\left(u_{m *}^{n} \mathcal{E}, \mathcal{G}\right) \cong \operatorname{Hom}_{\mathbf{D}\left(U_{n}\right)}\left(\mathcal{E}, u_{m}^{n !} \mathcal{G}\right) \quad\left(\mathcal{E} \in \mathbf{D}_{\mathrm{qc}}\left(U_{n}\right), \mathcal{G} \in \mathbf{D}\left(U_{m}\right)\right)
$$

(by which the natural map $u_{m *}^{n} u_{m}^{n !} \mathcal{G} \rightarrow \mathcal{G}$ corresponds to the identity map of $u_{m}^{n !} \mathcal{G}$ ) to reduce the commutativity question to that for a diagram of isomorphisms of functors from $\mathbf{D}_{\mathrm{qc}}^{+}\left(Y_{m}\right)$ to $\mathbf{D}_{\mathrm{qc}}\left(U_{n}\right)$ :

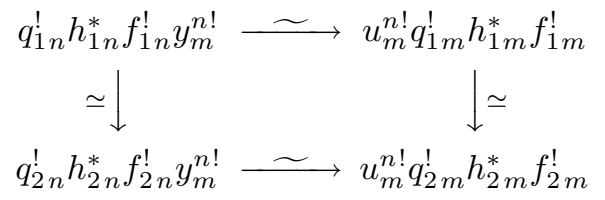

In this diagram only maps between ordinary schemes appear, so as before one can identify $h^{*}$ with $h^{!}$and define the vertical arrows via pseudofunctoriality. The horizontal arrows involve flat base change. However, under the identification of $h^{*}$

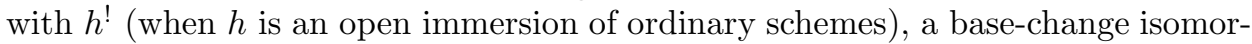
phism like $h_{1 n}^{*} x_{1 m}^{n !} \stackrel{\sim}{\longrightarrow} v_{1 m}^{n !} h_{1 m}^{*}$ becomes identified with the pseudofunctoriality isomorphism $h_{1 n}^{!} x_{1 m}^{n !} \stackrel{\sim}{\longrightarrow} v_{1 m}^{n !} h_{1 m}^{!}$. (See the above Digression.) With this in mind one finds again that pseudofunctoriality yields the desired commutativity.

This completes the proof of Lemma 1

Proposition 2 ([DFS], 8.3.1). Let there be given a commutative diagram

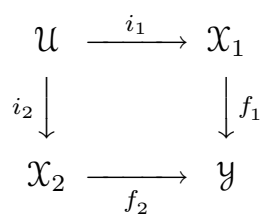

of noetherian formal schemes, with $f_{1}$ and $f_{2}$ pseudo-proper and $i_{1}$ and $i_{2}$ open immersions. Then one can define isomorphisms

$$
i_{1}^{*} f_{1}^{!} \mathcal{F} \stackrel{\sim}{\longrightarrow} i_{2}^{*} f_{2}^{!} \mathcal{F}, \quad i_{1}^{*} f_{1}^{\#} \mathcal{F} \stackrel{\sim}{\sim} i_{2}^{*} f_{2}^{\#} \mathcal{F} \quad\left(\mathcal{F} \in \widetilde{\mathbf{D}}_{\mathrm{qc}}^{+}(\mathrm{y})\right)
$$

such that the induced homology isomorphisms are canonical and functorial.

Proof. This is the particular case $q_{k}=$ identity, $h_{k}=i_{k}$, of Lemma 1.

Proposition 3 ([DFS], 8.3.2). If $f: X \rightarrow y$ is a pseudo-proper map of noetherian formal schemes, then

$$
f^{\#}\left(\mathbf{D}_{\mathrm{c}}^{+}(y)\right) \subset \mathbf{D}_{\mathrm{c}}^{+}(\mathcal{X})
$$

\footnotetext{
${ }^{3}$ In particular, "pseudofunctorial associativity": if $\gamma_{\psi, \varphi}:(\varphi \psi)^{!} \stackrel{\sim}{\longrightarrow} \psi^{!} \varphi^{!}$is the canonical isomorphism, then for any composition $\varphi \psi \chi$ it holds that $\left(\chi^{!} \gamma_{\psi, \varphi}\right) \circ \gamma_{\chi, \varphi \psi}$ and $\gamma_{\chi, \psi}\left(\varphi^{!}\right) \circ \gamma_{\psi \chi, \varphi}$ are the same isomorphism from $(\varphi \psi \chi)^{!}$to $\chi^{!} \psi^{!} \varphi^{!}$.
} 
Proof. As in loc. cit. we may assume that $y$ is affine, say $y=\operatorname{Spf}(A)$, and that $X$ can be covered by open subsets $j: \mathcal{U} \rightarrow X$ such that $\left.f\right|_{\mathfrak{U}}:=f \circ j$ factors as

$$
\mathcal{U} \stackrel{i}{\longrightarrow} \operatorname{Spf}(B) \stackrel{h}{\longrightarrow} \mathcal{P} \stackrel{p_{1}}{\longrightarrow} \operatorname{Spf}(A)
$$

where $i$ is a closed immersion, $h$ is an open immersion and $\mathcal{P}$ is the completion of the projective space $\mathbf{P}_{A}^{n}$ along some closed subset. Now for $\mathcal{F} \in \mathbf{D}_{\mathrm{c}}^{+}(y)$, Lemma 1 provides an isomorphism $j^{*} f^{\#} \mathcal{F} \cong i^{\#} h^{*} p_{1}^{\#} \mathcal{F}$, giving a reduction to the two cases (a) $f=p_{1}$ and (b) $f$ a closed immersion, cases dealt with at the end of the proof in loc. cit.

\section{REFERENCES}

[DFS] L. Alonso Tarrío, A. Jeremías López, J. Lipman, Duality and flat base change on formal schemes. Studies in duality on Noetherian formal schemes and non-Noetherian ordinary schemes, Contemporary Mathematics, 244, American Mathematical Soc., Providence, RI, 1999, 3-90. MR 2000h:14017

[Co] B. Conrad, Deligne's notes on Nagata Compactifications, item 5 on <http://www-math.

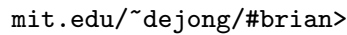

[De] P. Deligne, Cohomologie à supports propres. Théorie des Topos et cohomologie Étale des Schémas (SGA 4) Tome 3, Lecture Notes in Math. no. 305, Springer-Verlag, New York, 1973, 250-461. MR 50:7132

[Ha] R. Hartshorne, Residues and Duality, Lecture Notes in Math., no. 20, Springer-Verlag, New York, 1966. MR 36:5145

[HR] W. Heinzer and C. Rotthaus, Formal fibers and complete homomorphic images, Proc. Amer. Math. Soc. 120 (1994), 359-369. MR 94d:13020

[Li] J. Lipman, Non-noetherian Grothendieck duality. Studies in duality on Noetherian formal schemes and non-Noetherian ordinary schemes, Contemporary Mathematics, 244, American Mathematical Soc., Providence, RI, 1999, 115-123. MR 2000h:14017

[Lü] W. Lütkebohmert, On compactification of schemes, Manuscr. Math. 80, (1993), 95-111. MR 94h:14004

[Ne] A. Neeman, The Grothendieck duality theorem via Bousfield's techniques and Brown representability, J. Amer. Math. Soc. 9 (1996), 205-236. MR 96c:18006

[Ve] J. L. Verdier, Base change for twisted inverse image of coherent sheaves. Algebraic Geometry, Oxford Univ. Press, 1969, 393-408. MR 43:227

Departamento de Álxebra, Facultade de Matemáticas, Universidade de Santiago de Compostela, E-15782 Santiago de Compostela, Spain

E-mail address: leoalonso@usc.es

Departamento de Álxebra, Facultade de Matemáticas, Universidade de Santiago de Compostela, E-15782 Santiago de Compostela, Spain

E-mail address: jeremias@usc.es

Department of Mathematics, Purdue University, West Lafayette, Indiana 47907

E-mail address: lipman@math.purdue.edu 